\title{
Study Logistics Architecture for Grain Container Multimodal Transport Based on Multi-Agent
}

\section{Tingting Liu}

Software institute Dalian Jiaotong University

Dalian, 116024 ,China,

E-mail:2532659864@qq. com

\section{Guanghai Zheng}

Software Technology institute Dalian Jiaotong University

Dalian, 116024 ,China

E-mail:zhmos@163. com

In order to reduce the cost of grain container multimodal transport operations and improve collaborative management level of each link in grain container multimodal transport process, the paper presents an integrated business operation process and puts forward the architecture model of grain container multimodal transport system based on the Multi-Agent technology, further optimizing and integrating the management process of grain container multimodal transport operations. 


\section{Introduction}

The demand of small-lot transportations is increasing in modern grain logistics system. Container transport is a good way to meet the needs. Multimodal Transport is the key to coordinate the various transport modes.Different transportation ways are connected by Grain container intermodal transport which is more seamlessly.As a continuous, comprehensive and integrative grain transport, it can satisfy the needs of grain transportation especially in the long distance grain transportation.

Grain container multimodal transport has realized the seamless connection with different transportion ways. The research obtains many valuable achievements, Ma Caiwen designs the multimodal transport information system integrated with framework of virtual enterprise based on Multi-Agent[1];Zhou Minghua proposes to construct efficient linkages on the fully functional integrated service platform with the aim to provide the whole seamless information integration service for multimodal transport [2]; Shi Yan puts forward a combinational optimization model of intermodal transportation mode[3]; This paper uses Multi-Agent technology to research on grain container multimodal transport logistics system and optimizes the management process of grain container multimodal transport operations. The improved level of grain logistics management enables realize the enterprise to benefit from the grain container multimodal transport to the largest extent throughout the transport chain.

\section{Analysis Of Grain Container Multimodal Transport}

The container multimodal transport is a kind of transport organization forms for realizing the most benefit of container transport logistics. The container is taken as the transport unit anddifferent transportation ways are organically integrated together to form a continuous and comprehensive integration container transport. Container Multimodal Transport is a system that covers dynamic composition and operation. It consists of a multimodal transport operator (simply MTO) and the transport carrier. It coordinates and contacts each other with themultimodal transport to complete the task. MTO is responsible for the whole process of the transport, signing the multimodal transport and contracting with consignor. It's also responsible for performing or arranging for the performance via contracting with multimodal transport. The carrier holds both the the rights and obligations for the entire transport.Container multimodal transport operates different modes of transportation, insurance, customs and related government departments. The main business process of grain container intermodal transportation is shown in figure 1: Firstly, the grain dealer raises a grain container transport request to the MTO Secondly, they select a mode of transportation and the transport carrier in different regions. Thirdly, MTO submits the booking power of attorney to the shipping company includingshipper, consignee, loading port, unloading port and so on.Shipping company with good cabin to MTO issues modules receipt. MTO applies to railway carrier for railway transportation plan. The railway carrier sends a rail travel plan to MTO, the plan including the time, terminal, etc.. Fourthly, MTO releases information such as final destination and pick up time to the shipper/Finally, the grain customer completes grain container deliveryupon arrival at the destination. When the goods arrive, MTO notifies the consignee to pick up the goods and ends the grain container transport process. 


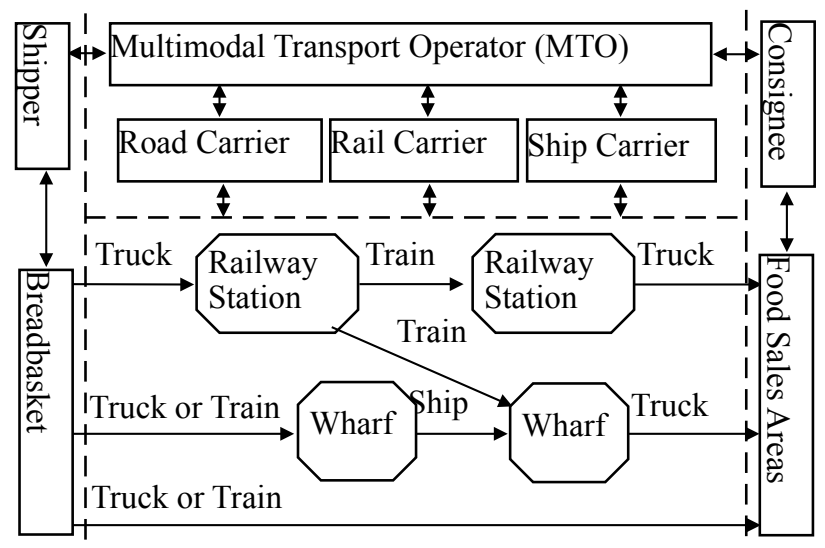

Figure 1: Business Process Diagram of Grain Container Multimodal Transport.

\section{The Agent Model for TheSystem of Grain Container Multimodal Transport}

\subsection{The Technology of Multi-Agent}

The agent is a software entity imitating human behavior while the Multi-Agent can imitate the social organization structure, division and cooperation.Agent owns the ability of perception and communication with outside. It also has the features of autonomy, interactivity, cooperation and adaptability, etc.Jennings concluded the applicability of the Agent [4]. Li Ying proposed the use of Multi-Agent in prediction and intelligent transportation[5].The research about MultiAgent on container transportation also achieves a good result. Davidson, P. proposed the transport system based on Agent as well[6]. Chen Bo proposed the transportation system with the Agent[7]. All of these researches have proven that the Agent can solve the problems in the transportation field. Multi-Agent system coordinates a group of independent intelligent Agent and predicts in advance.

\subsection{Constitution of TheSystem}

The grain container multimodal transport is a Multi-Agent system in which Container MTO is the core that cooperates partners. A whole Agent society includes operation Agent, resource Agent, interactive Agent,cooperative Agent and data service Agent, etc. The composition of system Agent is shown in figure 2:MTO-Agent receives the transportation project from the potential containers in different subsections which packages the external interaction and internal management behaviors. There are many transport means to be choosen.Consignor contacts with MTO. Consignor Agent packages stocking up and applies for transportation who takes delivery of the goods.Resource Agent simulates the transport carrier Agent which packages the food container transportation according to different factors. Each Agent of the system has different functions to achieving the whole transportation task of Multiunion transportation. In order to ensure the task to be performed successfully, the cooperation Agent needs to estimate the task proceeded by Agent. Therefore, before distributing the task to the professional Agents, it is needed to select suitable professional Agent to perform the task. After that,the task result should be fed back to thecooperation Agent. At last, the cooperation Agent gathers and deals with the results.Data service Agent provides a standard interface to access to the internal and external data source.The human-computer interactive Agent provides a graphical interface for users and facilitates to complete the interactive operation as soon as possible. 


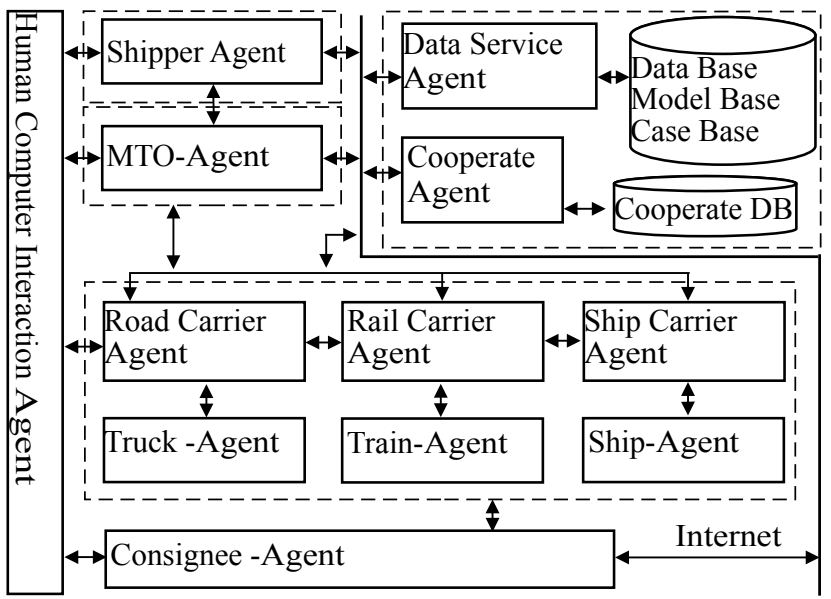

Figure 2:Structure of the Agent System

\section{The Cooperation Between Multi-Agents}

\subsection{Agent Cooperation}

The Multi-Agent system is mainly composed of autonomous Agents through interaction to solve complex problems [8]. In order to reduce the communication cost, the traffic and the resource consumption, The Agent history tracking sequence is proposed. The efficient information of each Agent is stored in the database, in order to enable the cooperative Agent to choose the appropriate collaboration object. Grain container multimodal transport needs to optimize the selection of different transport operators. Cooperative Agent needs to select the appropriate section of operator in the carriage of goods with least resources. It can decompose complex problems and choose different extents operators to resolve the grain container transportation problem.

\subsection{Agent Cooperation Model}

The cooperation model of the grain container multimodal transport uses four tuples $\mathrm{C}=$ $\{\mathrm{A}, \mathrm{E}, \mathrm{M}, \mathrm{P}\}$ to show the information, among which, A refers to the Agent collection, $\mathrm{M}$ refers to the communication between the Agent collaboration, receiving and sending module interactive messages; E refers to a set of events; P refers to a set of scripts used to control the rules of communication between the Agents. The event set in the Agent collaboration use six tuples $\mathrm{E}=\{\mathrm{Id}, \mathrm{Attr}, \mathrm{T}$, Pcon, A, Act $\}$ to show the time that event occurs; Pcon premises conditions of events; A events activation Agent group; Act is the Agent of the action set, including action planning action condition and action mode. It can perform a collection of actions.Agent is defined as four tuples: $\mathrm{A}=\{\mathrm{S}, \mathrm{Ty}, \mathrm{Dq}, \mathrm{Tq}\}$, among which $\mathrm{S}$ represents the current state of the Agent, a part of the mental model, and covers its own records, beliefs, desires and intentions of data; Ty refers to some type of Agent, specifically in the field of tasks; Dq meansthe target queue, defined as a set of variables. The set of variables calculate and compare the expected value $\mathrm{c}$ with the current value, making corresponding processingupon the result of comparison; $\mathrm{Tq}$ represnts partner Agent history information tracking queue, whichorganizes a local database among the Agent, and store the information such as the desire and ability, intention and action.By tracing the history information, andanalyzing desire and the desire of utility, system updates the collaborative assessment data dynamically. 
Collaboration among all the Agents is based on the grain container transport data requests. It requires the corresponding process to complete the data collection and processing. System interaction protocol with a standard FIPA request, Agent communication language (ACL) is used to define the message object.The message content is based on the grain container multimodal transport system ontology definition. Each Agent message model is based on the model, and the sender who determines the content of the message.

\subsection{Application of the Agent Cooperation}

Here is an example that how grain container multimodal transport logistics system interacts. As shown in figure 3,through the human-computer interaction Agent, the shipper Agent chooses MTO-Agent and makes a grain container transport request, MTO-Agent accepts the request and checks it before reaching an agreement. MTO-Agent makes a grain container transport request to cooperation Agent who smoothly completes different segments of cohesion. Eventually, the consignee Agent picks up the goods and finishs the grain container task.

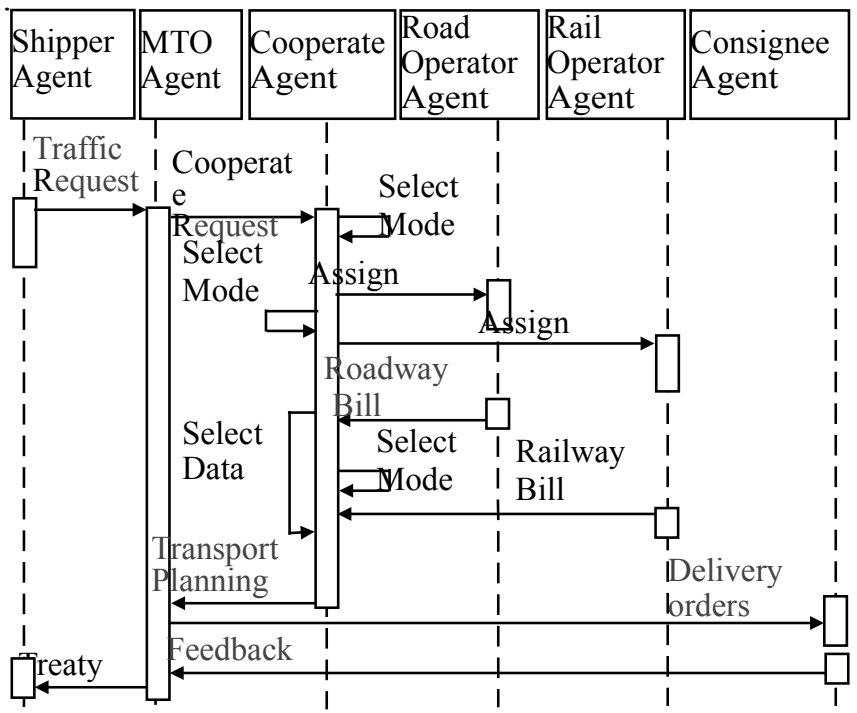

Figure 3:System Agent Interactions

\section{Applications}

The whole system is composed of 12 Agents distributed in three IBM servers, with the Linux operating system and Oracle $10 \mathrm{~g}$ database. Prototype of multi Agent system is widely applied in the development of JADE which is a pure JAVA, compatible with standard FIPA Agent platform, conforming to the framework of prototype system.

Examples of model application in transport network as shown in figure 4. Grain container is shipped from grain origin $\mathrm{C} 1$ to grain sales city $\mathrm{C} 8$, which goes through several cities. Urban transport mode can choose from road, rail or sea.

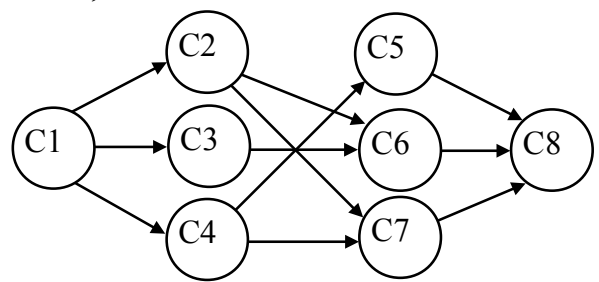

Figure 4:Grain Container Transport Path Graph 
Weight of each edge in the graph represent cities between different transportation modes of transportation cost and shipping time is shown in table 1 . The transfer viavarious modes of transportation costs and transit time is shown in table 2.

\begin{tabular}{|c|c|c|c|c|c|c|c|c|c|c|c|}
\hline & $\mathbf{c 1}$ & $\mathbf{c 1}$ & $\mathbf{c 1}$ & $\mathbf{c 2}$ & $\mathbf{c 2}$ & $\mathbf{c 3}$ & $\mathbf{c 4}$ & $\mathbf{c 4}$ & $\mathbf{c 5}$ & $\mathbf{c 6}$ & $\mathbf{c 7}$ \\
& - & - & - & - & - & - & - & - & - & - & - \\
& $\mathbf{c 2}$ & $\mathbf{c 3}$ & $\mathbf{c 4}$ & $\mathbf{c 6}$ & $\mathbf{c 7}$ & $\mathbf{c 6}$ & $\mathbf{c 5}$ & $\mathbf{c 7}$ & $\mathbf{c 8}$ & $\mathbf{c 8}$ & $\mathbf{c 8}$ \\
\hline 1 & 7 & 4 & 3 & 4 & 4 & 5 & 8 & 6 & 5 & 8 & 5 \\
& $\mathrm{~K}$ & $\mathrm{~K}$ & $\mathrm{~K}$ & $\mathrm{~K}$ & 4 & $\mathrm{~K}$ & $\mathrm{~K}$ & $\mathrm{~K}$ & $\mathrm{~K}$ & $\mathrm{~K}$ & $\mathrm{~K} /$ \\
& $/ 1$ & $/ 1$ & $/ 1$ & $/ 1$ & $/ 9$ & $/ 9$ & $/ 1$ & $/ 1$ & 1 & $/ 2$ & 9 \\
& 2 & 0 & 5 & 2 & & & 5 & 8 & 2 & 0 & \\
\hline 2 & 8 & 4 & 5 & 6 & 5 & 8 & 6 & 8 & 7 & 9 & 3 \\
& $\mathrm{~K}$ & $\mathrm{~K}$ & $\mathrm{~K}$ & $\mathrm{~K}$ & $\mathrm{~K}$ & $\mathrm{~K}$ & $\mathrm{~K}$ & $\mathrm{~K}$ & $\mathrm{~K}$ & $\mathrm{~K}$ & $\mathrm{~K} /$ \\
& $/ 1$ & $/ 1$ & $/ 1$ & $/ 1$ & $/ 1$ & $/ 1$ & $/ 1$ & $/ 1$ & $/ 1$ & $/ 1$ & 7 \\
& 0 & 2 & 3 & 7 & 0 & 5 & 3 & 5 & 6 & 5 & \\
\hline 3 & 4 & 1 & 4 & 3 & 3 & 4 & 5 & 4 & 6 & 4 & 2 \\
& $\mathrm{~K}$ & 1 & $\mathrm{~K}$ & $\mathrm{~K}$ & $\mathrm{~K}$ & $\mathrm{~K}$ & $\mathrm{~K}$ & $\mathrm{~K}$ & $\mathrm{~K}$ & $\mathrm{~K}$ & $\mathrm{~K} /$ \\
& $/ 1$ & $\mathrm{~K}$ & $/ 2$ & $/ 1$ & $/ 1$ & $/ 1$ & $/ 1$ & $/ 1$ & $/ 1$ & $/ 1$ & 14 \\
& 8 & $/ 9$ & 0 & 8 & 4 & 2 & 7 & 2 & 5 & 8 & 14 \\
\hline
\end{tabular}

Table 1:Transportation Costs That Between City And City/Transportation Time Table

Note: 1 Railway transportation, 2 Roadway Transportation, 3 seat transportation

\begin{tabular}{|c|c|c|c|c|c|c|}
\hline $\begin{array}{c}\text { Mode of } \\
\text { Transportatio } \\
\text { n }\end{array}$ & \multicolumn{2}{|c|}{$\begin{array}{c}\text { Railway } \\
\text { Transportatio } \\
\text { n }\end{array}$} & \multicolumn{2}{c|}{$\begin{array}{c}\text { Roadway } \\
\text { Transportatio } \\
\text { n }\end{array}$} & \multicolumn{2}{c|}{$\begin{array}{c}\text { Sea } \\
\text { Transportatio } \\
\text { n }\end{array}$} \\
\cline { 2 - 7 } & Costs & Time & Costs & Time & Costs & Time \\
\hline $\begin{array}{c}\text { Railway } \\
\text { Transportation }\end{array}$ & 0 & 0 & $1 \mathrm{~K}$ & 2 & $1 \mathrm{~K}$ & 2 \\
\hline $\begin{array}{c}\text { Roadway } \\
\text { Transportation }\end{array}$ & $1 \mathrm{~K}$ & 2 & 0 & 0 & $2 \mathrm{~K}$ & 1 \\
\hline $\begin{array}{c}\text { Sea } \\
\text { Transportation }\end{array}$ & $1 \mathrm{~K}$ & 2 & $2 \mathrm{~K}$ & 1 & 0 & 0 \\
\hline
\end{tabular}

Table 2: The Transit Shipment Costs Of Each Transportation Modes)/Transit Shipment Time Table

The prototype system uses the Dijkstra algorithm to solve problems with considerion of the cost and time limit. According to the different time demand, it can get the combination of the lowest shipping costs. Finally it can reach the following results: if the time is no more than 27 days, the path $\mathrm{C} 1-\mathrm{C} 2-\mathrm{C} 7-\mathrm{C} 8$ can be chosen. $\mathrm{C} 1-\mathrm{C} 2$ is the railway transportation while $\mathrm{C} 2-$ C7-C8 is the shipping transportation. The lowest cost is $16 \mathrm{~K}$; If the time is no more than 37 days, the path e C1-C4-C7-C8 can be selected.The mode of transportation is railway transport and the lowest cost is $13 \mathrm{~K}$. Prototype system realizes the grain container multimodal transport logistics system modeling and scheduling, With the coordination of each functional Agents grain container transportation is well controlled and managed.The different state of the Agent and the interactions between the Agents can be easily observed in JADE container. Furhtermore, it is more convenient for debugging and application, $t$ information sharing and work collaborations among different Agents.

\section{Conclusion}

Based on the analysis of the specific management process in grain container multimodal transport logistics system. Grain container multimodal transport logistics system framework is established.It studies the interactions among Agents and establishes an Agent interaction 
collaborative model. Through the verification of the system prototype, the model is valid, MTO can optimize the grain container multimodal transport operation process and achieve high integrated transport effiency with the choice of multimodal transport section operators.

\section{References}

[1] Ma Caiwen, Sun Guangqi. International multi-modal transport virtual enterprise information ntegration frame based on multi-agent. Journal of Dalian Maritime University. Vol.32, No.2, pp.7174, 2006.

[2] Shi Yan. Research and Implementation the Logistics Distribution System Based on Multimodal Transport. Journal of Microcomputer information. Vol.26, No.8, pp.215-222, 2010.

[3] Zhang Rong, Liu Zhiqi, Study on Container IntermodalTerm inal Information Platform. Journal of Tongji University (Natural Science). Vol.134, No.2, pp.201-206, 2006.

[4] Jennings, N.R. An Agent-Based Approach for Building Complex Software Systems. Communications of the ACM, Vol.44, No.4, pp.35-41, 2001.

[5] Li Ying.Application of Multi-Agent System in Forecasting and Intelligent Transportion System. Shanghai:East China University of Science and Technology Press,2004.

[6] Davidson, P., Henesey, L., Ramstedt, L., Törnquist, J. and Wernstedt, F., An analysis of agent based approaches to transport logistics, Transportation Research Part C: Emerging Technologies, Vol.13, No.4, pp.255-271, 2005.

[7] Chen Bo, Cheng HarryH. A Review of the Applications of Agent Technology in Traffic and Transportation Systems, IEEE Transactions on Intelligent Transportation Systems, Vol.11, No.2, pp.485-497, 2010.

[8] Luo Junmin, Zheng Shouqi, Zhong Lianjiong, Ontology and AORBCO Model, Journal of Microelectronics \& Computer, Vol.21, No.11, pp.33-36, 2004. 